\title{
Electron Microscopy and X-Ray Diffraction Analysis of Titanium Oxide Nanoparticles Synthesized by Pulsed Laser Ablation in Liquid
}

\author{
Jolanta Donėliené ${ }^{1}$, Matas Rudzikas ${ }^{1}$, Steffi Rades ${ }^{2}$, Ilona Dörfel², Burkhard Peplinski², Mario Sahre ${ }^{2}$, \\ Francesco Pellegrino $^{3}$, Valter Maurino ${ }^{3}$, Juras Ulbikas ${ }^{1}$, Algirdas Galdikas ${ }^{1}$ and Vasile-Dan Hodoroaba ${ }^{2}$ \\ 1. UAB "Modern E-Technologies", Vilnius, Lithuania. \\ 2. Federal Institute for Materials Research and Testing (BAM), Berlin, Germany. \\ 3. Department of Chemistry, University of Turin, Turin, Italy. \\ 4. Applied Research Institute for Prospective Technologies, Vilnius, Lithuania.
}

Numerous studies in the literature report on titanium dioxide nanoparticles as a harmless, cheap, facile, bio-compatible, chemically and mechanically stable and highly efficient photo-catalyst which can be exploited in many large-scale applications. One of the most promising techniques for the synthesis of titania nanoparticles in liquids or in polymeric matrices is pulsed laser ablation in liquids (PLAL), e.g. $[1,2]$. Thereby only a titanium plate and a 'green' solvent are the necessary materials.

The present paper investigates firstly whether the specific use of femtosecond lasers enables the control of the synthesized titanium oxide nanoparticles. Further, the morphology of the generated nanoparticles as well as their inner structure were analyzed by complementary electron microscopy techniques, i.e. SEM, TSEM and HR-TEM (applied as in [3]), and X-ray diffraction. The influence of the laser ablation time - as the main parameter, varied between $10 \mathrm{~min}$ and $90 \mathrm{~min}$ - has been quantified in terms of particles size distribution and crystalline structure.

The result of the variable laser ablation time is the generation of titanium dioxide nanoparticles of spherical shape with a diameter of most particles in the range of 10 to $20 \mathrm{~nm}$. The statistical evaluation of the particle size measurement demonstrates a rather constant mean particle size with increased laser ablation time, together with a decrease of size polydispersity up to 30 min laser ablation time, followed by a broadening of the moderate polydispersity with laser ablation times longer than 45 min. Further, the morphology of nanoparticle surface is either very smooth or provides asperities which are very similar with the 'rest' of co-generated nano-structured, irregularly-shaped material present outside the spherical particles on the sample substrate, see Figure 1. These findings hold true for the whole sequence of generated titanium oxide nanoparticles independent of laser ablation time. The morphology of the irregularly-shaped nano-debris seems to become more pronounced with longer laser ablation times, see Figure 1. EDX analysis on individual nanoparticles has confirmed that the main elements present in both spherical and irregularly-shaped particle components are only $\mathrm{Ti}$ and $\mathrm{O}$, see Figure 2.

As far as the crystalline structure of the particulate material generated by laser ablation is concerned, no clear distinction could be established between shorter and longer laser ablation times; the characterization of individual nanoparticles by HR-TEM, but also the integral analysis by X-ray diffraction have demonstrated the presence of non-phase-pure titanium oxide particles. A mix of fully (poly-)crystalline, partially crystalline as well as amorphous particles has been identified as not being strongly dependent on the laser ablation time. Further, the nano-structured material component complementing the spherical particles has been proven to be of crystalline nature.

Additionally, ICP-MS analysis have been carried out on a series of eight samples obtained after 
increasing laser ablation time, so that it was possible to determine the generation rate of nano-particulate titanium oxide of about $0.07 \mathrm{mg} / \mathrm{min}$.

\section{References:}

[1] SM Hong et al, Bull Korean Chem Soc 34 (No. 1) (2013), p. 279.

[2] SI Alnassar et al, Optics \& Laser Technology 51 (2013), p. 17.

[3] N Wollschläger et al, Mater Charact 131 (2017), p. 39.

[4] This work was supported by the SETNanoMetro Seventh Framework Programme project (project number 604577; call identifier FP7-NMP-2013_LARGE-7).
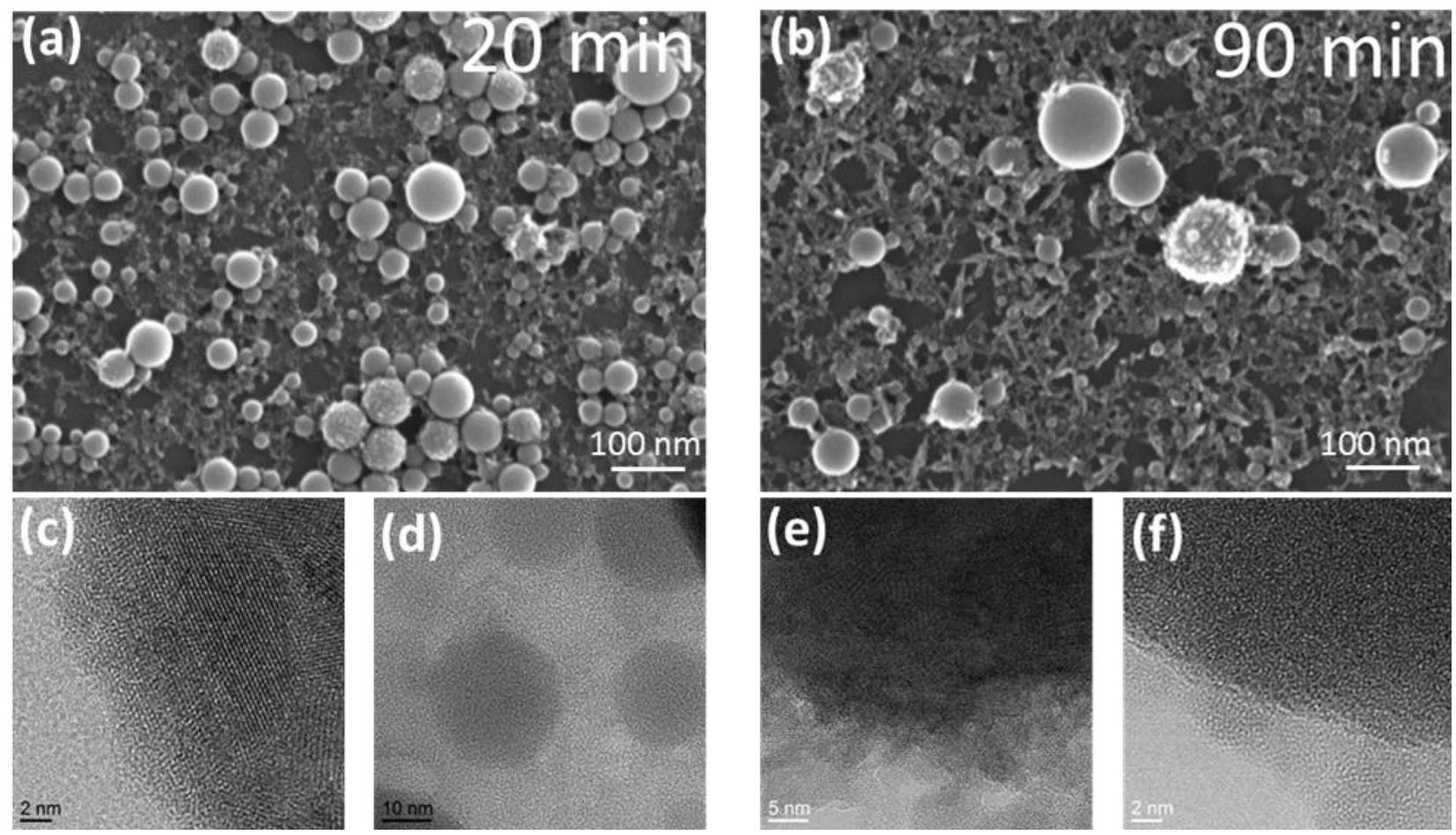

Figure 1. SEM (top) and HR-TEM (bottom) images of $\mathrm{Ti}_{\mathrm{x}} \mathrm{O}_{\mathrm{y}}$ nanoparticles after $20 \mathrm{~min}$ (left) and 90 min (right) duration of laser ablation. Further parameters of laser ablation: $260 \mathrm{fs}, 513 \mathrm{~nm}, 4.9 \mathrm{~W}, 330$ $\mathrm{kHz}, 15 \mu \mathrm{J}, 330 \mathrm{~mm} / \mathrm{s}$.

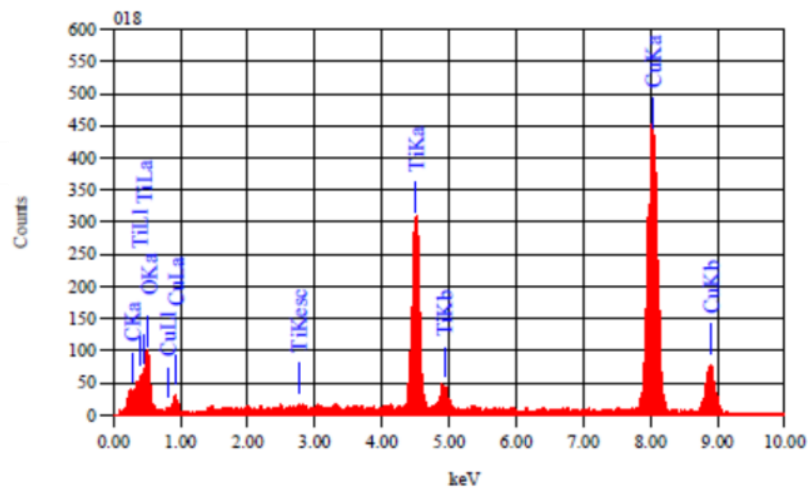

Figure 2. Point EDX spectrum taken on a rough particle as that TEM imaged in Figure 1(e). $\mathrm{Cu}$ and $\mathrm{C}$ signals come from the sample support (lacey Carbon film). 